Diabetologe 2011 · 7:471-471

DOI 10.1007/s11428-011-0752-6

(c) Springer-Verlag 2011

\author{
E. Siegel \\ St. Vincenz Krankenhaus, Limburg
}

\title{
Diabetesversorgung im Krankenhaus
}

men sollte die Versorgung von Patienten mit Diabetes mellitus als Hauptdiagnose vornehmlich in zertifizierten Einrichtungen der Deutschen Diabetes-Gesellschaft (DDG) als zuständiger Fachgesellschaft erfolgen, denn diese weisen die notwendige Struktur- und Prozessqualität auf. Gleiches gilt für die Behandlung des diabetischen Fußsyndroms in entsprechend hierfür qualifizierten und zertifizierten Einrichtungen.

Die Behandlung eines Diabetes mellitus als Nebendiagnose sollte eigentlich in allen Krankenhäusern erfolgen. Für die Diabetesbehandlung im Krankenhaus müssen die besonderen Gegebenheiten berücksichtigt werden, die den unterschiedlichen Anforderungen, einschließlich Stresssituationen der Patienten, zeitweiligem Nahrungsentzug, enteraler oder parenteraler Ernährung, Medikamenteninteraktionen z. B. mit Kortison, und vielen anderen Besonderheiten Rechnung tragen. Aufgrund der weiten Verbreitung betrifft dieses sämtliche nichtoperativen und operativen Fächer.

Die hierfür notwendige Struktur- und Prozessqualität wurde von der Fachgesellschaft bislang nicht definiert. Hier besteht dringender Handlungsbedarf. Stringente Prozesse zur Erkennung, Erfassung, Betreuung und Abrechnung von Patienten mit Diabetes mellitus sind nur in wenigen Klinken etabliert, und die medizinischen Belange der Patienten werden nicht erfüllt.

Angesichts des hohen Anteils Betroffener unter den stationären Patienten könnte deren erneute Wahl eines Krankenhauses auch davon abhängen, wie auf das spezielle Problem des Diabetes mellitus in der jeweiligen Klinik eingegangen wurde.
Die Publikationen in diesem Heft sollen die Sensibilität für das Thema Diabetes im stationären Bereich schärfen und auf spezifische Fragestellungen im $\mathrm{Zu}$ sammenhang mit der Versorgung von Patienten mit Diabetes mellitus in deutschen Krankenhäusern hinweisen. In einigen Schwerpunktbeiträgen kann allerdings keinesfalls die komplette stationäre Diabetologie abgebildet werden.

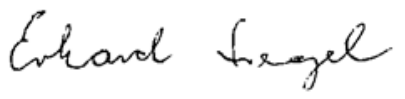

E. Siegel

\section{Korrespondenzadresse PD Dr. E. Siegel}

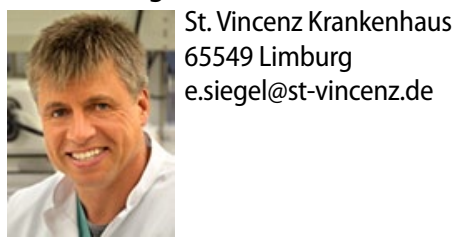

